\title{
Uptake of Cystatin by Melanoma Cells in Culture
}

\author{
Lauren Deady ${ }^{1}$, James L. Cox ${ }^{2 *}$ \\ ${ }^{1}$ Department of Biology, Truman State University, Kirksville, USA \\ ${ }^{2}$ Department of Biochemistry, AT Still University, Kirksville, USA \\ Email: ${ }^{*}$ jcox@atsu.edu
}

Received March 8, 2013; revised April 7, 2013; accepted April 21, 2013

Copyright (C) 2013 Lauren Deady, James L. Cox. This is an open access article distributed under the Creative Commons Attribution License, which permits unrestricted use, distribution, and reproduction in any medium, provided the original work is properly cited.

\begin{abstract}
The cystatins are a super family of cysteine protease inhibitors which are ubiquitous in their biologic occurrence. Cystatin C, a type II cystatin, is primarily a secreted protein found in most biological fluids. Besides acting as inhibitors of cathepsin, the cystatins have been found to have some non-inhibitor related functions and multiple physiological roles. Much interest has been generated for the cystatins as metastasis "suppressor-like" proteins, as they have been shown to inhibit metastasis for multiple cancer types. The sites and actions of the cystatins related to tumor suppressor actions are still unclear, however. In this work, we have examined the uptake of cystatin by metastatic melanoma cells in culture. Our results indicate cystatin uptake is mediated by a non-canonical endocytotic pathway in B16 murine melanoma cells.
\end{abstract}

Keywords: Cystatin; Melanoma; Endocytosis; Fluorescent

\section{Introduction}

The cystatins are a super family of cysteine protease inhibitors that primarily inhibit cathepsin-type cysteine proteases (family C1) [1]. The cystatins serve to block excess or inadvertent cysteine protease activity that may occur in cells or tissues. Additional physiological roles for the cystatins are expanding and include inhibition of microbial cell invasion and replication, modulation of immune function, and growth factor effect son cells [2-4]. The type I cystatins are primarily intracellular proteins. However, low levels of extra cellular type I cystatins have also been reported and they may therefore influence other cells in the vicinity of the cystatin producing cells [5]. Type II cystatins are primarily secreted inhibitor proteins, which may also have some intracellular actions as uptake by cellular endocytosis has been observed in vivo and in cells in culture [6]. As secreted inhibitors, they may interact with a wide variety of different cell types. For instance, uptake of cystatin $\mathrm{C}$ into neuroretinal ganglion cells has recently been reported [7]. Cystatins are able to suppress the metastasis of a variety of cancer cell types when over expressed by cancer cells $[8,9]$. Although a number of studies have examined changes in cystatin over-expression effects on cancer cells, exact sites of cellular action of the cystatins are still unclear [10-12]. The cellular sites of cystatin action are particularly important as cystatins have recently been shown to

${ }^{*}$ Corresponding author. not only interact with non-cathepsin proteins but also influence gene expression in cancer cells [13,14]. As many cancers types over-express and secrete cathepsins, which are also found plasma membrane bound and even in nuclei, there are both extra cellular and intracellular sites where cystatins may interfere with cathepsin activity and function in tumor cells [15]. To explore questions related to cystatin sites of action and cellular entry we have examined the process of cystatin uptake by murine melanoma cells in cell culture.

\section{Materials and Methods}

\subsection{Cell Culture and Antibodies}

B16F10 melanoma cells were purchased from the ATCC. Cells were cultured at $37^{\circ} \mathrm{C}$ at $5 \% \mathrm{CO}_{2}$ in RPMI 1640 medium (Atlanta Biological, Inc.) containing 10\% fetal bovine serum and antibiotics (100 I.U./ml penicillin, 100 $\mu \mathrm{g} / \mathrm{ml}$ streptomycin, and $0.25 \mu \mathrm{g} / \mathrm{ml}$ amphotericin ) (MP Biomedicals). Caveolin-1 polyclonal antibody was purchased from Santa Cruz Biotechnology, Inc. and ezrin mouse monoclonal antibody was from DSHB, University of Iowa, Iowa City, Iowa. Anti-Cystatin C polyclonal antibody was purchased from Thermo Scientific.

\subsection{FITC Labeling and Uptake Conditions}

Egg white chicken cystatin (Calbiochem) was labeled with FITC for cell uptake experiments. Labeling of cys- 
tatin $(100 \mu \mathrm{g})$ was carried out in $100 \mu \mathrm{l}$ of $50 \mathrm{mM}$ $\mathrm{Na}_{2} \mathrm{CO}_{3}, 0.85 \% \mathrm{NaCl}$ buffer containing $0.8 \mathrm{mM}$ FITC (Sigma-Aldrich) on a tube rotator for 1 hour at $4 \mathrm{oC}$. Following the reaction, the cystatin solution was dialyzed overnight against 1 liter of phosphate buffered saline (PBS) at $4^{\circ} \mathrm{C}$. B16F10 melanoma cells were seeded on acidpre-treated glass coverslips overnight. FITC-labeled cystatin $(5 \mu \mathrm{l}(\sim 5 \mu \mathrm{g}$ cystatin) per $50 \mu \mathrm{l}$ phenol red-free RPMI media plus $10 \%$ FBS) was added directly onto cells on coverslips and incubation followed for 1 hour at $37^{\circ} \mathrm{C}$ under cell culture conditions. The cells were then washed $3 \times$ with PBS and fixed with ice cold methanol for 10 minutes at $-20^{\circ} \mathrm{C}$. In some experiments, $5 \mu 1$ Texas Red dextran, $1 \mathrm{mg} / \mathrm{ml}$ (Molecular Probes, Eugene, Oregon) was added per $50 \mu \mathrm{l}$ media at the same time as labeled cystatin with parallel incubation conditions as for labeled cystatin. Inhibitors were obtained from SigmaAldrich and used to pretreat the cells as follows: Chlorpromazine pretreatment was for 1 hour at $50 \mu \mathrm{M}$ before cystatin uptake. Blebbistatin pretreatment was for 1 hour at $100 \mu \mathrm{M}$ before cystatin uptake. Colchicine and wortmannin were used at $50 \mu \mathrm{M}$ and $100 \mathrm{nM}$ respectively in 1 hour pretreatment before cystatin uptake experiments.

\subsection{Time Course of Cystatin Uptake into Melanoma Cells}

Melanoma cells were plated in 96 -well plates at $2 \times 104$ cells/well and allowed to attach overnight. FITC-labeled labeled cystatin was added to the cells at 1:25 dilution in culture medium for various times. Labeled cystatin solution was then removed and cells were washed with PBS. The plate was then read with a BioTek Fl $\times-800$ plate reader for recording of the relative fluorescence of the wells.

\subsection{Fluorescent Antibody Staining}

The method followed for fluorescent antibody staining was from Chan et al. [16]. B16F10 melanoma cells were attached to glass coverslips overnight under cell culture conditions. After cystatin uptake protocol, cells were fixed for 10 minutes with cold methanol at $-20^{\circ} \mathrm{C}$. Cells were then washed twice with PBS and permeabilized with $0.1 \%$ Triton $\times 100$ in PBS for 2 minutes followed by three additional washes with PBS. Permeabilized cells were then blocked with 5\% horse serum in PBS for 30 minutes. Primary antibodies (1:100 diluted in blocking solution) were spotted on parafilm in $50 \mu \mathrm{l}$ drops and the coverslips were inverted onto drops for 30 minutes. Following primary antibody, coverslips were washed 3 times for 5 minutes each with PBS. Fluorescent labeled secondary antibodies were then applied as for primary with incubation for 30 minutes at room temperature. Three PBS washes were again applied, and a brief rinse with deionized water was carried out before mounting the coverslips on glass slides with Prolong Gold mounting medium (Invitrogen).

\subsection{Fluorescent and Confocal Microscopy}

Fluorescent microscopy was imaged with a Zeiss Axiovert $200 \mathrm{M}$ microscope $(63 \times$ oil with 1.4 NA Plan apo lens and an Axiocam MRM camera. Images were captured with Axiovision version 4.7 software. Confocal microscopic analysis and image capture was with a Leica SP5 microscope using a $63 \times$ oil objective (Leica Microsystems, Heidelberg, Germany).

\section{Results and Discussion}

Cystatins are synthesized by virtually all eukaryotic cells, and the type II cystatins are secreted cysteine protease inhibitors that are found in all body fluids at various levels [17]. As cystatins are the major cysteine protease inhibitors found in biological fluids, it was of interest to examine the interaction and uptake of cystatin into cells in culture. The levels of cystatin present in serum, for example, might be taken up by endothelial cells and influence the behavior of these cells. The uptake of many different labeled proteins by cells in culture has been examined. However, there are very few reports on the process of cystatin uptake in metastatic cancer cells. Our goal in this study was to probe the process of cystatin uptake in a metastatic cancer cells because overexpression of cystatin in cancer cells or delivery of cystatin to cancer cells in vivo has been shown to be effective in blocking metastasis [18]. We wished to probe the pathway of cystatin uptake in cancer cells through the use of inhibitors and antibody labeling of potential cellular uptake components.

The direct uptake of labeled cystatin from cell culture media into metastatic murine B16F10 melanoma cells as a model system was investigated. We focused on B16F10 melanoma because our previous work showed cystatin $\mathrm{C}$ could significantly inhibit metastasis in mice when overexpressed in these cells [8]. The type II cystatins are found in all biological fluids and therefore are able to interact and be taken up by cells these fluids come into contact with. Fluorescently-labeled (FITC) chicken cystatin was prepared and added to melanoma cells in culture such that cellular uptake could be imaged with fluorescent microscopy. We added the FITC-labeled chicken egg white cystatin to B16 melanoma cells for a brief period of time (10 minutes) and found pretreatment of the cells with unlabeled cystatin could partially block cystatin uptake by the cells (Figure 1). This result suggested a potential receptor mediated uptake of cystatin by the melanoma cells. A time course of cystatin uptake by B16 melanoma cells showed a rapid (minutes) cell associated cystatin fluorescence followed by a lower level fluores cence by 30 minutes (Figure 2). We next wanted to look 


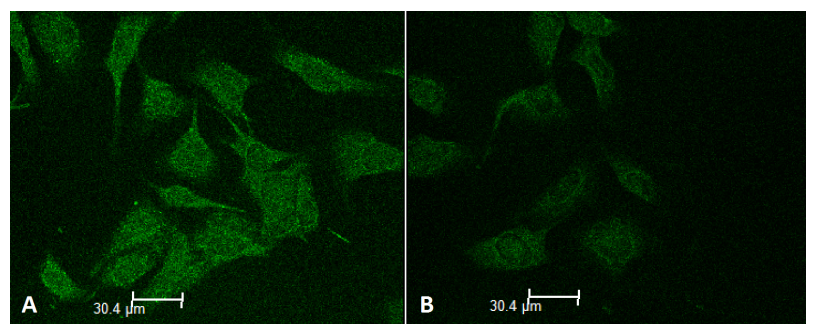

Figure 1. Uptake of FITC-labeled cystatin into B16F10 melanoma cells. Cells were incubated with FITC-labeled cystatin for 10 minutes, washed twice with PBS, fixed with methanol, mounted on coverslips for confocal microscopy. (A) without or (B) with unlabeled cystatin $(20 \mathrm{ug} / \mathrm{ml})$ pretreatment of cells.

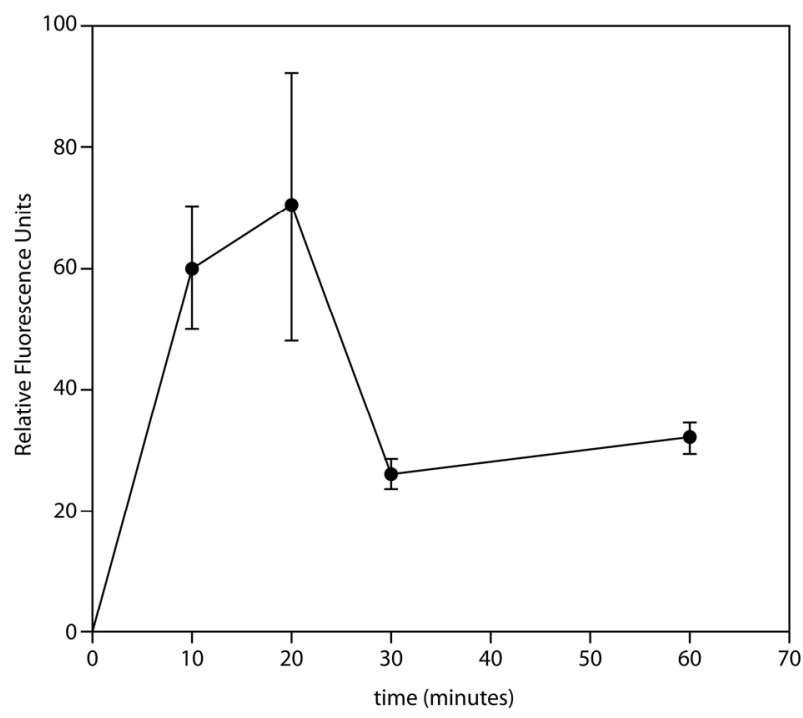

Figure 2. Time course of cystatin uptake into B16 melanoma cells. FITC-labeled cystatin was added to B16F10 melanoma cells for various times. Following timed cystatin exposure, the cells were washed with PBS and fluorescence was measured with a fluorescent plate reader. Relative fluorescence was recorded +/- SEM for each time point.

at cystatin uptake under different conditions and test certain inhibitors for their actions on cystatin uptake by the melanoma cells. When chicken cystatin was added to B16F10 melanoma cells for 60 minutes under cell culture conditions there was uptake of cystatin into cytoplasmic vesicles which ranged in cellular location from general cytoplasmic to perinuclear (Figure 3). Exposure of the cells to Texas Red-labeled dextran together with the FITC-labeled cystatin appeared to label distinct intracellular vesicle populations (Figure 3). This observation suggested that each fluorescent label followed a distinct endocytotic pathway and that uptake of cystatin is not simply macropinocytosis as has been described for dextran particle endocytosis. It was also noted that perinuclear regions of the melanoma cells tended to show colocalization of both labeled cystatin and dextran. Since lysosomes are generally perinuclearin location and repre-
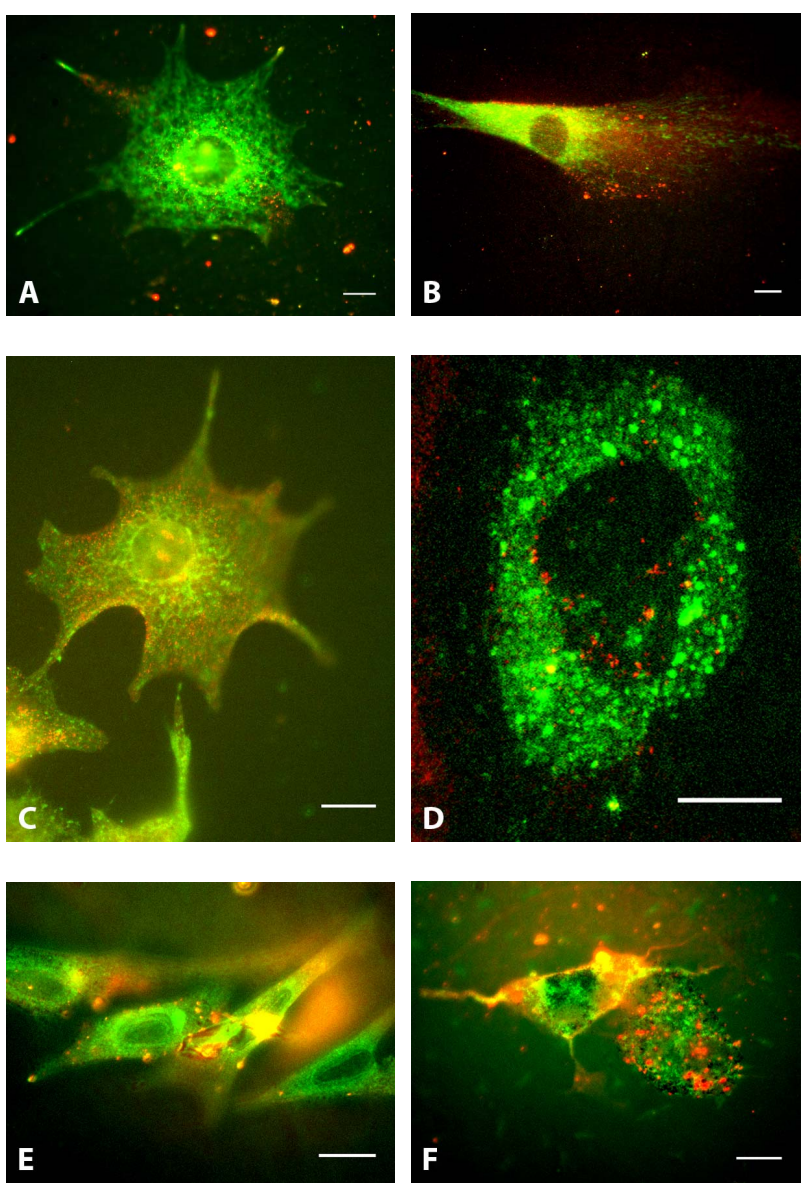

Figure 3. Uptake of FITC-labeled cystatin into B16F10 melanoma cells.Melanoma cells were incubated with both labeled cystatin and Texas red dextran beads for one hour at $37^{\circ} \mathrm{C}$. Cells were then methanol fixed and in some cases stained with fluorescent antibody. ((A), (B)) control uptake of cystatin (green) and dextran (red); (C) cystatin uptake (green) plus caveolin-1 stained (red); (D) same as c, plus additional $2 \times$ magnification; (E) control cystatin uptake (green) and dextran (red); (F) chlorpromazine pretreated for 1 hour. Representative images of cystatin uptake experiments performed in triplicate. Scale bar $=15 \mu \mathrm{m}$.

sent a final destination for many endocytosed proteins, it appears the bulk of the endocytosed cystatin is destined for lysosomes.

A major endocytotic pathway is through caveolae mediated uptake [19]. To examine this possibility, we used a primary antibody to caveolin- 1 and a secondary TRITClabeled antibody to stain cells which had been fixed and permeabilized following one hour exposure to labeled cystatin. We found no co-localization of labeled cystatin vesicles and caveolin-1 in the melanoma cell periphery near the membrane region (Figure 3). This finding suggests uptake of cystatin is not mediated by caveosomes or caveolin-1 associated vesicles. To examine potential clathrin mediated uptake of cystatin, we pre-incubated melanoma cells in culture with chlorpromazine, a known 
inhibitor of clathrin mediated uptake [20]. We found no inhibition of cystatin uptake with chlorpromazine treatment, suggesting cystatin uptake is non-clathrin mediated (Figure 1).

The involvement of the cellular cytoskeleton in endocytosis is well known. We tested the involvement of myosin II in the uptake of cystatin by pretreatment of the cells with blebbistatin, a specific inhibitor of non-muscle myosin [21]. No major inhibition of labeled cystatin uptake was observed with blebbistatin pretreatment of the melanoma cells (Figure 4). In separate experiments, colchicine, a microtubule inhibitor, was used to pre-treat cells prior to cystatin uptake experiments. No dramatic inhibition of cystatin uptake was noted with colchicine pretreatment (Figure 4). We did not expect inhibition with colchicine as other reports found vesicular trafficking of macropinocytosis most closely linked to microtubules. Since our earlier dextran uptake showed macropinocytosis was not involved the lack of colchicine effect was not surprising. We did find partial inhibition of cystatin uptake with wortmannin inhibition of phosphoinositide 3-kinase (PI3K) (Figure 4). It was noted that intra-
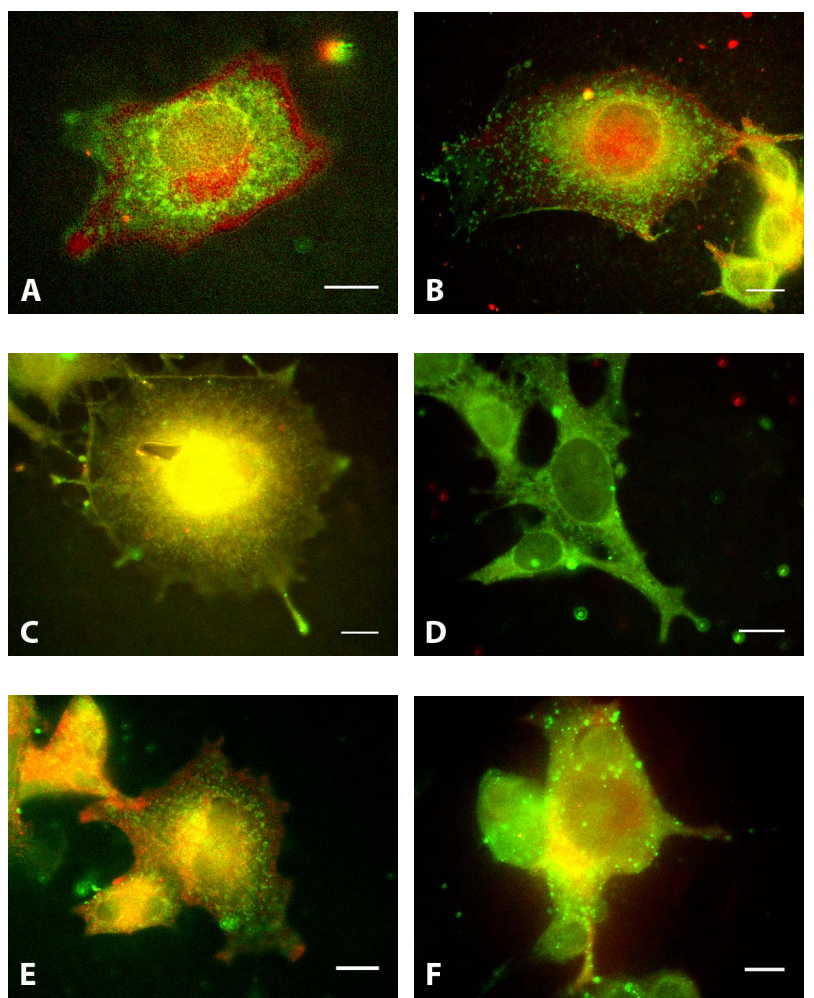

Figure 4. Uptake of FITC-labeled cystatin into B16F10 melanoma cells, inhibitor treatment. Melanoma cells were pre-incubated at $37^{\circ} \mathrm{C}$ for 1 hour prior to labeled-cystatin uptake experiments. (A) control blebbistatin; (B) blebbistatin treated; (C) control colchicines; (D) colchicine treated; (E) control wortmannin; (F) wortmannin treated. Representative images of cystatin uptake experiments performed in triplicate. Scale bar $=15 \mu \mathrm{m}$. cellular vesicles were enlarged which might indicate PI3K inhibition interferes with endocytotic vesicle trafficking. PI3K is involved in macrophage and other cell phagocytosis through $\mathrm{Fc} \gamma \mathrm{R}$ receptors [22]. Although of interest, more work will have to be undertaken to look at possible involvement of phagocytosis through a receptor mediated pathway in melanoma cells.

Researchers have described another phagocytic pathway in melanoma and some other cancer cell types through ezrin linked vacuoles [23]. This phagocytic pathway is dependent on the actin cytoskeleton, and vacuoles found in this pathway also express lamp1, which might be expected for lysosomal destination of the vacuole cargo. Our approach to test for ezrin-linked cystatin phagocytosis was to allow melanoma cell uptake of labeled cystatin and then stain the cells for ezrin. We found no clear evidence of ezrin co-localization with fluorescent cystatin which had been taken up (Figure 5). Future work should also examine lamp1 and rab5 which are endosomal markers linked with ezrin, particularly in light of the blockade of metastasis seen with ezrin mutants seen in metastatic melanoma [24]. In this work we have focused on a general description of the cystatin uptake process in metastatic melanoma cells. Information on the pathway of cystatin uptake and utilization by cancer cells may lead to new ways to exploit influence of cystatinas an anti-metastatic agent in the multiple cancer types previously studied.

\section{Conclusion}

Work shown here on cystatin and other recent work had shown that uptake of cystatin by cancer cells is rapid, selective, and saturable, all pointing towards an active, specific receptor mediated process [25]. First, we have demonstrated the bulk of labeled cystatin uptake by melanoma cells is not through macropinocytosis. We have also extended previous work to show that cystatin uptake by melanoma cells is mediated by a non-clathrin and non-caveolin endocytotic pathway. The uptake of cystatin into melanoma cells requires neither microtubule involvement nor myosin II dependence, but the process showed some inhibition with wortmannin treatment,

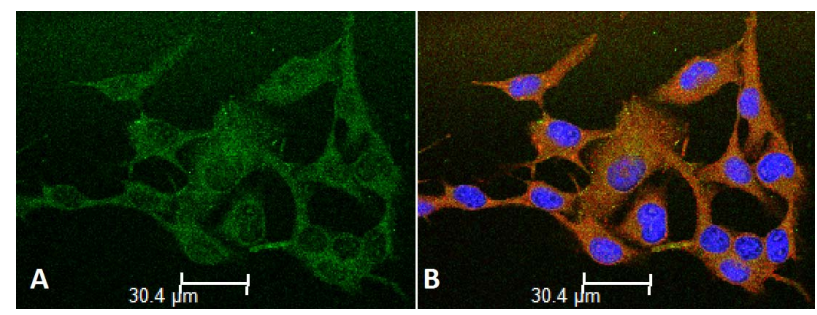

Figure 5. Confocal image of labeled cystatin uptake (green) plus anti-ezrinantibody stained (red) B16 melanoma cells. (A) cystatin uptake, green channel, (B) cystatin uptake and anti-ezrin (red secondary). Nuclear stain (blue), Draq5. 
suggesting PI3 kinase involvement. Ezrin staining did not localize with cystatin $\mathrm{C}$ uptake vesicles. Future work will define the membrane receptor for cystatin, define further requirements for cystatin uptake, and examine more closely possible involvement of phagocytic pathways for cystatin uptake in metastatic cells.

\section{REFERENCES}

[1] V. Turk, V. Stoka and D. Turk, "Cystatins: Biochemical and Structural Properties, and Medical Relevance," Frontiers in Bioscience: A Journal and Virtual Library, Vol. 13, 2008, pp. 5406-5420.

[2] M. F. Blankenvoorde, W. van't Hof, E. Walgreen-Weterings, T. J. van Steenbergen, H. S. Brand, E. C. Veerman and A. V. N. Amerongen, "Cystatin and CystatinDerived Peptides Have Antibacterial Activity against the Pathogen Porphyromonas gingivalis," Biological Chemistry, Vol. 379, No. 11, 1998, pp. 1371-1375.

[3] N. Kopitar-Jerala, "The Role of Cystatins in Cells of the Immune System," FEBS Letters, Vol. 580, No. 27, 2006, pp. 6295-6301. doi:10.1016/j.febslet.2006.10.055

[4] Q. Sun, "Growth Stimulation of 3T3 Fibroblasts by Cystatin," Experimental Cell Research, Vol. 180, No. 1, 1989, pp. 150-160. doi:10.1016/0014-4827(89)90219-X

[5] M. Abrahamson, A. J. Barrett, G. Salvesen and A. Grubb, "Isolation of Six Cysteine Proteinase Inhibitors from Human Urine. Their Physicochemical and Enzyme Kinetic Properties and Concentrations in Biological Fluids," The Journal of Biological Chemistry, Vol. 261, No. 24, 1986, pp. 11282-11289.

[6] H. Wallin, M. Bjarnadottir, L. K. Vogel, J. Wassélius, U. Ekström and M. Abrahamson, "Cystatins-Extra- and Intracellular Cysteine Protease Inhibitors: High-Level Secretion and Uptake of Cystatin C in Human Neuroblastoma Cells.," Biochimie, Vol. 92, No. 11, 2010, pp. 16251634. doi:10.1016/j.biochi.2010.08.011

[7] J. Wassélius, K. Johansson, K. Håkansson, M. Abrahamson and B. Ehinger, "Cystatin C Uptake in the Eye," Graefe's Archive for Clinical and Experimental Ophthalmology = Albrecht von Graefes Archiv für klinische und experimentelle Ophthalmologie, Vol. 243, No. 6, 2005, pp. 583-592.

[8] H. Ervin and J. L. Cox, "Late Stage Inhibition of Hematogenous Melanoma Metastasis by Cystatin C Over- Expression," Cancer Cell International, Vol. 5, No. 1, 2005, p. 14. doi:10.1186/1475-2867-5-14

[9] W. Li, F. Ding, L. Zhang, Z. Liu, Y. Wu, A. Luo, M. Wu, M. Wang, Q. Zhan and Z. Liu, "Overexpression of Stefin A in Human Esophageal Squamous Cell Carcinoma Cells Inhibits Tumor Cell Growth, Angiogenesis, Invasion, and Metastasis," Clinical Cancer Research: An Official Journal of the American Association for Cancer Research, Vol. 11, No. 24, 2005, pp. 8753-8762.

[10] S. D. Konduri, N. Yanamandra, K. Siddique, A. Joseph, D. H. Dinh, W. C. Olivero, M. Gujrati, G. Kouraklis, A. Swaroop, A. P. Kyritsis and J. S. Rao, "Modulation of Cystatin C Expression Impairs the Invasive and Tumori- genic Potential of Human Glioblastoma Cells," Oncogene, Vol. 21, No. 57, 2002, pp. 8705-8712.

doi:10.1038/sj.onc.1205949

[11] B. Wegiel, T. Jiborn, M. Abrahamson, L. Helczynski, L. Otterbein, J. L. Persson and A. Bjartell, "Cystatin C Is Downregulated in Prostate Cancer and Modulates Invasion of Prostate Cancer Cells via MAPK/Erk and Androgen Receptor Pathways," PloS One, Vol. 4, No. 11, 2009, e7953. doi:10.1371/journal.pone.0007953

[12] S. Alvarez-Díaz, N. Valle, J. M. García, C. Peña, J. M. P. Freije, V. Quesada, A. Astudillo, F. Bonilla, C. LópezOtín and A. Muñoz, "Cystatin D Is a Candidate Tumor Suppressor Gene Induced by Vitamin D in Human Colon Cancer Cells.," The Journal of Clinical Investigation, Vol. 119, No. 8, 2009, pp. 2343-2358. doi:10.1172/JCI37205

[13] J. P. Sokol and W. P. Schiemann, "Cystatin C Antagonizes Transforming Growth Factor Beta Signaling in Normal and Cancer Cells," Molecular Cancer Research: MCR, Vol. 2, No. 3, 2004, pp. 183-195.

[14] J. Song, C. Jie, P. Polk, R. Shridhar, T. Clair, J. Zhang, L. Yin and D. Keppler, "The Candidate Tumor Suppressor CST6 Alters the Gene Expression Profile of Human Breast Carcinoma Cells: Down-Regulation of the Potent Mitogenic, Motogenic, and Angiogenic Factor Autotaxin," Biochemical and Biophysical Research Communications, Vol. 340, No. 1, 2006, pp. 175-182. doi:10.1016/j.bbrc.2005.11.171

[15] D. Cavallo-Medved and B. F. Sloane, "Cell-Surface Cathepsin B: Understanding Its Functional Significance," Current Topics in Developmental Biology, Vol. 54, 2003, pp. 313-341. doi:10.1016/S0070-2153(03)54013-3

[16] K. T. Chan, C. L. Cortesio and A. Huttenlocher, "Integrins in Cell Migration," Methods in Enzymology, Vol. 426, 2007, pp. 47-67. doi:10.1016/S0076-6879(07)26003-3

[17] J. Ochieng and G. Chaudhuri, "Cystatin Superfamily," Journal of Health Care for the Poor and Underserved, Vol. 21, No. 1, 2010, pp. 51-70. doi:10.1353/hpu.0.0257

[18] J. L. Cox, "Cystatins and Cancer," Frontiers in Bioscience: A Journal and Virtual Library, Vol. 14, 2009, pp. 463-474.

[19] G. Bathori, L. Cervenak and I. Karadi, "Caveolae-An Alternative Endocytotic Pathway for Targeted Drug Delivery," Critical Reviews in Therapeutic Drug Carrier Systems, Vol. 21, No. 2, 2004, pp. 67-95. doi:10.1615/CritRevTherDrugCarrierSyst.v21.i2.10

[20] K. M. Hussain, K. L. J. Leong, M. M.-L. Ng and J. J. H. $\mathrm{Chu}$, "The Essential Role of Clathrin-Mediated Endocytosis in the Infectious Entry of Human Enterovirus 71," The Journal of Biological Chemistry, Vol. 286, No. 1, 2011, pp. 309-321. doi:10.1074/jbc.M110.168468

[21] M. Rey, A. Valenzuela-Fernández, A. Urzainqui, M. Yáñez-Mó, M. Pérez-Martínez, P. Penela, F. Mayor and F. Sánchez-Madrid, "Myosin IIA Is Involved in the Endocytosis of CXCR4 Induced by SDF-1alpha.," Journal of Cell Science, Vol. 120, No. 6, 2007, pp. 1126-1133.

[22] M. Bohdanowicz, G. Cosío, J. M. Backer and S. Grinstein, "Class I and Class III Phosphoinositide 3-Kinases Are Required for Actin Polymerization That Propels Phago- 
somes," The Journal of Cell Biology, Vol. 191, No. 5, 2010, pp. 999-1012. doi:10.1083/jcb.201004005

[23] L. Lugini, P. Matarrese, A. Tinari, F. Lozupone, C. Federici, E. Iessi, M. Gentile, F. Luciani, G. Parmiani, L. Rivoltini, W. Malorni and S. Fais, "Cannibalism of Live Lymphocytes by Human Metastatic but Not Primary Melanoma Cells.," Cancer Research, Vol. 66, No. 7, 2006, pp. 3629-3638. doi:10.1158/0008-5472.CAN-05-3204

[24] C. Federici, D. Brambilla, F. Lozupone, P. Matarrese, A. de Milito, L. Lugini, E. Iessi, S. Cecchetti, M. Marino, M.
Perdicchio, M. Logozzi, M. Spada, W. Malorni and S. Fais, "Pleiotropic Function of Ezrin in Human Metastatic Melanomas," International Journal of Cancer, Vol. 124, No. 12, 2009, pp. 2804-2812.

[25] U. Ekström, H. Wallin, J. Lorenzo, B. Holmqvist, M. Abrahamson and F. X. Avilés, "Internalization of Cystatin C in Human Cell Lines," The FEBS Journal, Vol. 275, No. 18,2008 , pp. 4571-4582. doi:10.1111/j.1742-4658.2008.06600.x 